\title{
Method for Increasing the Energy Efficiency of Wirelessly Networked Ambulatory Health Monitoring Devices
}

\author{
Bruce Moulton*1, Graham Croucher $^{1}$, Andrew Varis ${ }^{12}$, June Chen ${ }^{12}$ \\ *1Corresponding author Faculty of Engineering and Information Technology \\ ${ }^{2}$ Faculty of Science, University of Technology Sydney (UTS), Sydney, Australia \\ brucem@eng.uts.edu.au \\ doi: 10.4156/jcit.vol5.issue1.1
}

\begin{abstract}
In-home healthcare applications that use wearable devices ordinarily have strict power constraints due to the small size of the battery in the device. The power constraints are a key driver of research to develop new methods for improving the energy efficiency of ambulatory health monitoring devices. The radiocommunication components typically consume a large proportion of the available energy in systems such as these. Given that radio transmissions use far more power than on-board processing, it is proposed that energy can be conserved by performing fall detection at the node. The proposed algorithm is intended to be performed at the node and provide a suitable balance between power consumption and detection accuracy. The research and prototype system described in this article focuses on wearable fall detection devices to be used elderly people who are living in non-hospital settings, and discusses considerations arising from the development of a prototype system. The outcomes of the system design and development process are discussed, and conclusions are drawn concerning the potential of the method to improve the energy efficiency of fall detection systems.
\end{abstract}

\section{Keywords}

eHealth, falls, energy, home, sensor, trauma, ambulatory monitoring, aged care.

\section{Introduction}

The aging population together with changing economic circumstances has seen hospital and nursing aged-care facilities come under increasing pressure in recent years. An emerging trend is the increasing adoption of sensing and communication technologies that enable aged people to remain safely in their own homes. Such technologies include in-home systems for detecting medical emergencies that make use of wirelessly networked sensor-transceiver devices. In some systems that are currently being developed, fixed sensing devices are positioned in high-risk locations such as bathrooms and stairs. There are other systems that use wearable devices, or a combination of fixed and wearable devices.

Advances in ultra-low-power wearable sensors and transceivers are leading to the convergence of technologies which extend to the implementation of integrated wireless on-body systems that provide realtime sensing and interpretation capabilities. In some cases, sensor hardware is to be worn on the body or woven into fabric/clothing, and in other cases, it is to be implanted inside the body. The recent developments have opened the door to new possibilities for applications in healthcare, medical diagnostics, disaster management, environmental and habitat monitoring and emergency response $[1,2,3]$.

Costs of the associated components are rapidly decreasing, and the hardware is rapidly becoming smaller and more power-efficient. Advances have also been seen in battery technologies, but the constrained power sources continue to provide a significant challenge, so there is considerable motivation to explore various ways of reducing energy consumption.

Wearable healthcare sensor nodes are preferably small, lightweight and able to be worn without inconvenience to the user. This requirement severely limits the amount of energy available to the device over its lifetime, as the need for portability dictates that the power source remains small and independent [4]. Despite recent forays into renewable energy sources, the power source often takes the form of a lightweight battery, making energy a critically limited resource, one which should be conserved wherever possible [5]. Thus there is a driver for improved energy efficiency in both body area networks (BANs) and wireless sensor networks (WSN). The radio-communication components of BANs and WSN typically consume a large proportion of the available energy. Each byte transmitted by the radio consumes as much power as 8000 instructions on the microprocessor [1]. The radio 
typically accounts for more than $50 \%$ of the overall system energy budget $[4,5,6,7]$. Thus efforts directed towards increasing the overall energy efficiency (and hence longevity) have increasingly focused on ways of reducing the amount of energy consumed by radio transmissions.

Wirelessly-networked wearable health monitoring systems present specific design-challenges with respect to energy consumption. For example, an accelerometer and a transceiver can be used to monitor and broadcast changes in a person's physical activities which could be indicative of a fall. If not treated quickly, a fall is twice as likely to be fatal, hence the value of fall detection technology [8]. Given the potential for such systems to greatly improve elderly people's safety, research is directed towards improving the accuracy and efficiency of these systems.

\section{Reducing Energy Consumption}

Three issues relevant for improving the energy efficiency of the radio components are discussed as follows.

\subsection{Path Loss}

It is well understood that movement presents specific problems for wireless local area networks (WLANs). Body area networks (BANs) typically experience even more movement than WLANs, and it is difficult to construct an accurate model the effect of this movement on a channel.

The human body is partially conductive, and different elements (e.g. skin, bone, blood) have very different dielectric constants and impedances. Absorption varies with frequency and the characteristics of the tissue $[9,10,11,12]$. In addition, different parts of the body, for example, the head, arms, legs and torso, have very different overall thicknesses. Further, the geometry of different people's bodies varies widely from one to another. A small child affects path loss in significantly different ways from those of a large adult. Path loss in BANs can vary considerably due to different placements of the transceivers and different postures and movements of the body.

Given that the critical issues relating to shadowing and path loss in BANs are quite different from those of WLANs and non-body WSNs, it seems likely that different approaches to power control may be appropriate for BANs $[13,14,15]$. This being the case, it appears there are good reasons for exploring BAN- specific transmission power-level control schemes as a potential way to achieve power savings while taking path-loss into account in BANs.

\subsection{Cross layer approach}

It has been proposed that energy efficient design in such circumstances requires a cross-layer approach [5]. A cross-layer approach may entail the combined consideration of the Physical (PHY), Medium Access Control (MAC) and Network layers.

At the lowest layers, it may require the consideration of the specifications and configuration of the wireless radio, for example, the transmission power level. At the middle layers, it concerns the protocols which govern access to the shared wireless medium for each device. At the higher layers, it necessitates the optimization of the routing or movement of data between the nodes in the network as the data heads towards its final destination.

It can be difficult to delineate various layers, particularly when algorithm and protocol solutions already implement cross-layer approaches. Nevertheless, it is widely recognized that cross-layer optimization can minimize significant energy wastage and hence provide longer network lifetime. Given that this is the case, the potential for cross-layer approaches is of considerable interest for efforts to improve the energy efficiency.

\subsection{Transmission power control}

Reliable communication between wireless radios can be ensured by transmitting at the maximum transmission power available. However, this leads to energy wastage as the radio may operate just as reliably at lower transmission levels. Transmission power is difficult to set effectively as varying this usually affects delay, bit error rate, network capacity, interference and network topology [16]. Increasing a nodes' transmission power increases the range of transmission, allowing contact with a greater number of neighboring nodes. This strengthens network connectivity, enabling data to require fewer hops to reach its destination at the cost of greater power consumption. However, this also increases the amount of network interference (and therefore delays), decreasing network bandwidth [16].

With heterogeneous or small to medium scale homogenous networks, transmission power can be effectively combined with appropriate MAC protocols 
to ensure optimal connectivity without a significant energy or computational overhead.

TP control methods can be separated into three levels; network, node and packet level solutions [17]. The Network level, the coarsest solution, provides one transmission power for the entire network. These solutions do not use the variable transmission powers of individual wireless radios, and lead to significant energy overheads. Often this is the case with traditional wireless connectivity [17]. Examples of algorithms at the network level include the Fixed Transmission Power algorithm (FTP) and the Equal Transmission Power algorithm (ETP). Both of these algorithms use different techniques for determining the node transmission power, with FTP generally using the maximum and ETP using the minimum for satisfactory connectivity. With static, uniformly distributed nodes, ETP may be effective, but in all other cases, these algorithms lead to inefficient use of energy. They also require global information for implementation [18].

Node level solutions provide one transmission power for all neighbors connected to that specific node. The Diverse Transmission Power algorithm (DTP) is an example of such and finds a minimum power for individual nodes to establish network connectivity. Global information is required for this algorithm; the network may establish asymmetric communication links, and be unable to respond to a node if transmission power is set low due to another node being closer [18]. Other examples include the Local Mean Algorithm (LMA) and Local Mean of Neighbors algorithm (LMN), which work on the premise that each node needs a certain level of node connections. LMA uses broadcasting to determine the number of direct neighbors in range and alters transmission power accordingly to sit within a predetermined threshold. LMN uses a similar method but also determines the mean number of nodes that its neighbors are connected to, further optimizing connectivity [18].

Packet level solutions provide controllable levels of transmission power depending on the quality of the link between two nodes. They use packet metrics to determine the current state of the link and whether actions are required to optimize this link. The Adaptive Transmit Power Control (ATPC) algorithm is an example of this. ATPC uses the Received Signal Strength Indicator (RSSI) and Link Quality Indicator (LQI) metrics in a closed feedback loop between paired nodes to ensure both signal strength and signal quality are maintained at appropriate levels. This algorithm initially uses beacon signals at each signal level to populate a neighbor table and form a predictive model and consequently uses notifications sent by the receiving node to indicate whether modification of transmission power level is required on a packet by packet basis [17].

The Practical Transmit Power Control scheme is another algorithm designed for use at the packet level. This focuses on using a weighted mean of the RSSI values to control whether the transmission power should be altered between two communicating nodes [7]. This algorithm is slower to react than ATPC but the benefit of this over the ATPC algorithm is the lack of initialization phase and the smaller amount of computation required per packet.

In considering each of these different forms of power control, it is necessary to take into account the computational and communicational overheads associated with finer control. At the coarser, Network level, we sacrifice finer control for less overheads. In a well-designed, static system, this approach may be more effective. As we gain finer and finer control over the transmission power, this generally invokes a higher cost in computation and in communicating the necessary metrics. However, this fine control may in fact lead to a longer network lifetime in wireless sensor networks with roaming or asymmetric nodes. It is also necessary to consider the functions of the network. For example, the demands placed on a network by a fall detection application are very different from those of a heart rate monitoring application.

\section{Falls}

One third of falls among the elderly is in the home environment [19]. There are many factors contributing towards falls in the elderly. These include things like wet surfaces, changes in floor levels (stairs), dimly lit areas around the house, deteriorating sensory factors of the individual and climbing or having to reach to high levels. The complexity of the problem raises the need for a robust algorithm that is able to detect falls, and several of the key considerations are discussed in the following sections.

\subsection{Sideways falls tend to be more dangerous than forwards falls}

Most falls are forwards, but the majority of fallrelated fatalities are caused by sideways falls. A study of 14 different residential care facilities for the elderly over the course of 18 months recorded all falls experienced by 680 residents. The study recorded the 
timing, daily activities, direction of fall and severity. The findings indicated that $63 \%$ of the falls observed resulted in injury, most commonly during the day, when the elder was most active. The study also found that even though most activity is in the living areas, certain areas of the house are appear to be more dangerous than other areas; it was found that people are more likely to fall in the bathroom than in the dining room [20].

A study by Hayes et al [21] conducted research using younger subjects because it was deemed risky to experiment with older individuals given potential wrist, hip or vertebral fractures and joint sprains. This study was the first to address involuntary falls in the forwards and backwards directions. The study examined fall directions and pelvis impact location due to four disturbances (faint, slip, step down, trip) at three gait speeds (fast, normal and slow) in young adults who were instructed not to attempt recovery, but to fall passively all the way to the ground once disturbed. The study focused more on falls impacting on the hip and pelvic region and the associated injuries, and found that at fast gait speed, slips and faints commonly resulted in forward falls with frontal impacts leading to abdominal pelvis impacts. In contrast, as the gait speed decreased, slips typically resulted in falls in the sideways or backwards direction, impacting the hip or buttocks, and faints caused a higher number of sideways falls, impacting near the hip.

Studies such as these are of great value not only for healthcare providers and caregivers, but also for engineers who are seeking to develop fall detection systems. The research highlights the need to account for variables including gait speeds, the use of walking aids, and low impact falls, and research in this area is used to inform the development of methods for detecting falls discussed in the following section.

\subsection{Fall detection}

In-home or residential care fall detection can be accomplished by wearable devices, fixed devices, or a combination of both. Fixed devices include cameras, infrared sensors and pressure mats. Such devices are widely used in residential care or retirement settings, but are less prevalent in the home situation. Wearable devices include tilt sensors, accelerometers and gyroscopes. Accelerometers for fall detection have been the subject of prior research, and currently provide good balance between performance and cost.
An accelerometer-based algorithm to detect falls must distinguish between falls and daily living activities. A study by Bourke et al [22] proposes a fall detection algorithm that used a tri-axial accelerometer to discriminate between a fall event and daily living activities. The algorithm uses experimentallydetermined upper and lower fall threshold values obtained by positioning the accelerometer on the thigh and trunk. Other studies suggest that the waist and the small of the back are optimum on-body locations for an accelerometer [e.g. 23]; moving limbs creates a lot of variance in acceleration values if the accelerometer is attached to a limb. The algorithm proposed in [22] analyzes the resultant signal from the tri-axial accelerometer, and relies on the fact that when stationary, the root-sum-of-squares value from the accelerometer is a constant $+1 \mathrm{~g}$.

Reference [23] describes in detail three different algorithms called Conjoined Angle Change and Magnitude Detection, Simple Magnitude Detection and Advanced Magnitude Detection. The basis of these algorithms is to detect a large change in acceleration from the initial orientation, above an acceleration magnitude threshold. The system then analyses the orientation of the user to confirm whether the large acceleration change was really a fall. Considerations relating to these three algorithms are summarized in Table 1.

Table 1. Classes of fall detection algorithm

\begin{tabular}{|c|c|}
\hline $\begin{array}{c}\text { Conjoined } \\
\text { Angle Change } \\
\text { and } \\
\text { Magnitude } \\
\text { Detection } \\
\text { algorithm }\end{array}$ & $\begin{array}{l}\text { This class of algorithm observes } \\
\text { changes in angle orientation of the } \\
\text { user while making comparison of a } \\
\text { set threshold acceleration value } \\
\text { within the same time interval. When } \\
\text { an angle change is flagged, this } \\
\text { initiates the algorithm observation so } \\
\text { that when coupled by a large } \\
\text { acceleration within the same time } \\
\text { frame, this identifies a fall. However, } \\
\text { further algorithm improvement was } \\
\text { necessary as it did not take into } \\
\text { account that large accelerations and } \\
\text { angle change are common in } \\
\text { activities of daily living (ADL), such } \\
\text { as picking something up from the } \\
\text { floor, stretching, sitting down or } \\
\text { getting up from bed. }\end{array}$ \\
\hline $\begin{array}{l}\text { Simple } \\
\text { Magnitude } \\
\text { Detection } \\
\text { algorithm }\end{array}$ & $\begin{array}{l}\text { This class of algorithm observes a } \\
\text { large change in the acceleration from } \\
\text { the initial orientation, waits until the } \\
\text { acceleration ends, analyzes the } \\
\text { orientation, and if it is determined }\end{array}$ \\
\hline
\end{tabular}




\begin{tabular}{|c|c|}
\hline & $\begin{array}{l}\text { that the user was on the ground for an } \\
\text { extended period of time or } \\
\text { horizontally positioned on it, a fall is } \\
\text { identified. Continual analysis of the } \\
\text { user's orientation is necessary for this } \\
\text { algorithm to function. During testing, } \\
\text { this algorithm correctly detected the } \\
\text { majority of falls with a noticeable } \\
\text { change in the user's acceleration } \\
\text { magnitude, resulting in the user lying } \\
\text { horizontally on the ground. However, } \\
\text { a limitation of this model was that no } \\
\text { fall would be predicted if the user fell } \\
\text { and was not horizontal to the ground. } \\
\text { The model does not take into account } \\
\text { that the user will try to hold on to an } \\
\text { object such as a bed, table or wall as } \\
\text { he or she is falling, resulting in the } \\
\text { user inclined or slumped next to the } \\
\text { object. }\end{array}$ \\
\hline $\begin{array}{l}\text { Advanced } \\
\text { Magnitude } \\
\text { Detection } \\
\text { algorithm }\end{array}$ & $\begin{array}{l}\text { This algorithm was developed due to } \\
\text { the limitation found in the Simple } \\
\text { Magnitude Detection algorithm. The } \\
\text { algorithm was amended to } \\
\text { compensate for this. The initial } \\
\text { magnitude and position analysis was } \\
\text { kept the same; however the special } \\
\text { cases were accounted for with further } \\
\text { position analysis. This algorithm } \\
\text { functions to observe a large change in } \\
\text { the acceleration, wait until the large } \\
\text { acceleration ends, analyze orientation } \\
\text { and if the orientation revealed the } \\
\text { user to be horizontal with the ground, } \\
\text { or in the relative inactivity the user } \\
\text { was still not upright, whether on an } \\
\text { inclination or not, classify this as a } \\
\text { fall. }\end{array}$ \\
\hline
\end{tabular}

An algorithm presented in [24] (based on [22]) is a simplified version of the Advanced Magnitude Detection (AMD) algorithm. In [23], an AMD analyses the user's orientation and determines whether the user's body is horizontal to the ground; if yes, it is classified as a fall, and if not, the user has "orientation designated as deviating from uprightness". The algorithm gives the user a certain amount of time to become accustomed again. The sum vector of the triaxial accelerometer signals is more accurate in fall detection than single-axis thresholds [23].

Haux et al [24] start their algorithm by waiting for a large acceleration. This is recognized by the use of a magnitude vector/threshold value. When the large acceleration ends, the fall should be over even if it is an indirect fall and the algorithm senses more than one magnitude. The algorithm then saves the position of the person and analyses it for a time slice.

This approach can take angled falls into account by using a time slice function where three things may happen: the saved position (initial) differs greatly from the new position, another large acceleration occurs or nothing happens or no change occurs with the saved position.

According to [24], the first case refers to normal fall-free activity, looping and restarting the fall detection algorithm. The second case occurs when there is a subsequent fall and the algorithm starts at the point where it waits until the acceleration ends. The final and third case causes an alarm and reports a fall.

An implication of this is that the algorithm should be reasonably capable of identifying falls which result in the person landing in sitting or other orientation, and not just falls that result in the user lying horizontally on the floor.

It has been proposed that accuracy may be reduced when monitoring a large acceleration [23]; one approach is to accurately analyze each of the $\mathrm{x}, \mathrm{y}$ and $\mathrm{z}$ axes individually, whereas another is to simply analyze a "magnitude vector" which combines all three axes as follows:

$$
r=\sqrt{x^{2}+y^{2}+z^{2}}
$$

The magnitude vector can detect large accelerations in all directions, allowing detection of falls on each side of the body with the same method. The study suggests that there is no significant difference in accuracy between the two types of analyses using the Simple Magnitude Detection algorithm.

Another study undertaken validate the data collection of a new fall detector prototype and to define the sensitivity and specificity of three different fall detection algorithms (each of increasing complexity) with simulated falls from 20 middle-aged subjects aged 40-65 years old [25]. The activities of daily living performed by the middle-aged subjects living at home together with 21 people aged 58-98 years living in a residential care unit were used as a reference only. One of the research difficulties is that it is undesirable to request older people to undertake intentional falls. The results from the study indicated a sensitivity of $97.5 \%$ and specificityy of $100 \%$ was achieved when differentiating various types of falls. 


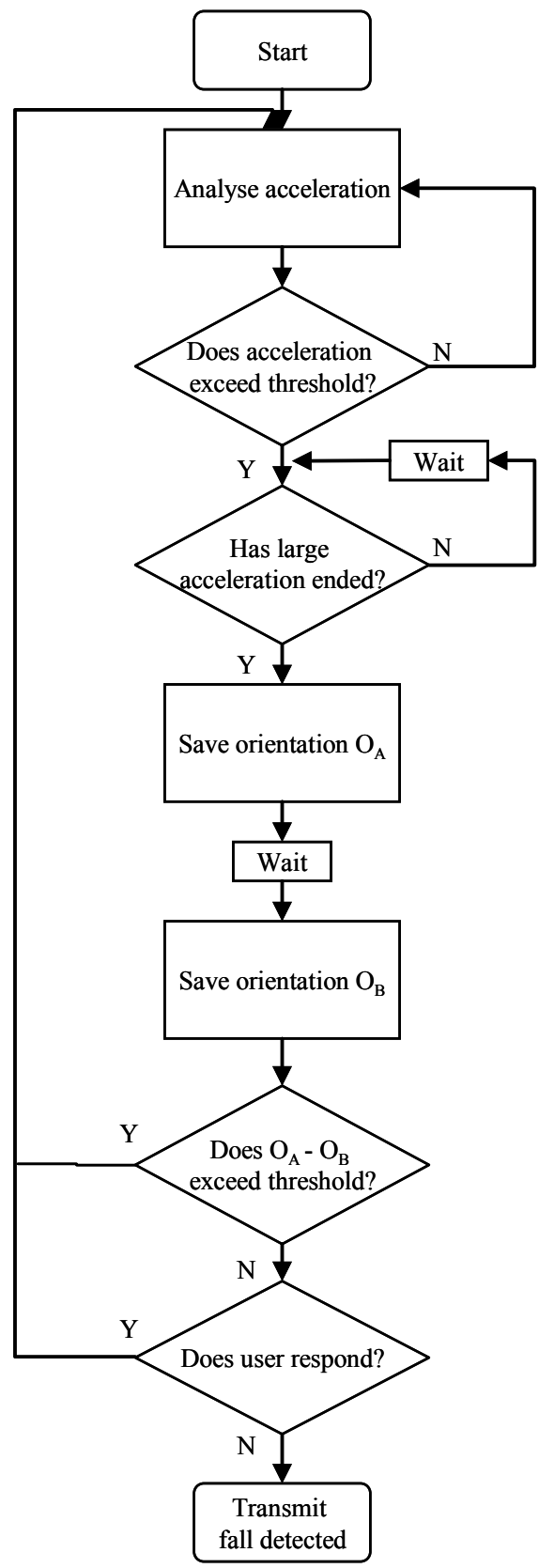

Figure 1. Algorithm implemented at the sensor node in the prototype system

The algorithm proposed here (Figure 1) is functionally similar to that given in [24]. It is proposed that this algorithm should be suitable for detection of all types of falls, not just falls resulting in the user horizontal on the floor, and that it should be suitable for a LIS302DL ultra-compact low-power digital output 3-axis accelerometer worn on the waist paired with a small low power data acquisition- microcontroller-transmitter system on a chip such as the CC981H Sensium.

In the given application context the algorithm works as follows. Consider an elderly man who trips over the first step on his flight of stairs at home. His body experiences a large acceleration in the forward direction, causing him to be inclined on the stairs. The "large acceleration" has ended, but he is not horizontal due to the slope of the stairs. The following may occur:

a. He picks himself up (and the algorithm re-starts; the fall is not severely incapacitating hence not transmitted); if he falls a second time, another large acceleration is detected

b. His position remains unchanged for the next few minutes; in this case the difference in orientation $\left(\mathrm{O}_{\mathrm{A}}\right.$ minus $\left.\mathrm{O}_{\mathrm{B}}\right)$ would remain below a pre-set threshold, and the system would then request a response from the user to determine whether the user is incapacitated. If the user does not respond, the fall is transmitted

If a variable transmission power protocol is used, the "fall-detected" message is transmitted at full power. In this way, it is proposed that the detection of falls may occur at the node, and in so doing, minimize accelerometer-related radio transmissions within the BAN and reduce consumption of energy. The falldetected message is accompanied by the raw data relating to the large acceleration and subsequent lack of movement, so that further analyses of the data may be performed prior to initiation of an alert or call for help.

\section{Conclusions}

Energy constraints are a primary concern for onbody wireless fall detection systems. Given that radio transmissions use far more power than on-board processing, it is proposed that energy can be conserved by performing some of the fall detection at the node. This has the effect of greatly reducing the length and frequency of broadcasts from the node.

One of the key issues relevant for improving the energy efficiency of the radio components includes the need for improved models to accurately model path loss through and around a moving human body. Crosslayer approaches and transmission power control approaches are also worthy of further consideration. One of the limitations of power control is that a higher cost in computation and in communicating the necessary metrics is incurred by processes which attempt to gain finer control over the transmission 
power level. However, it is thought that finer control may in fact lead to a longer network lifetime in wireless sensor networks or body area networks with roaming or asymmetric nodes.

It is also necessary to consider the specific functions of the system. For example, a blood oxygenation or heart rate monitoring application has energy requirements that differ greatly from those of a fall detection application. Further, when designing systems to detect falls, it is necessary to consider differences in the types of falls people experience, and differences in the requirements of various users of the systems. Another consideration is that falls to the side are more dangerous than falls to the front, and an implication of this is that a higher false positive rate may be accepted if the fall is to the side.

The experience of designing and implementing the prototype system was very valuable for identifying and understanding the many and complex considerations to be taken into account when attempting to design a system which has an acceptable balance between energy efficiency and detection accuracy.

\section{References}

[1] Culler, D., Estrin, V., Srivastava, M. 2004, 'Overview of Sensor Networks', Computer, vol. 37, no. 8, pp. 4149.

[2] Moulton, B., Chen, J., Croucher, G., Lal, S., Lawrence, E., Mahendran, L., Varis, A. (2009) Ambulatory health monitoring and remote sensing systems to be used by outpatients and elders at home: user-related design considerations. Proceedings of the 11th IEEE International Conference on e-Health Networking, Applications \& Services, IEEE Healthcom 2009, 16-18 December 2009.

[3] Moulton, B, Chaczko, Z., Karatovic, M. (2009) Updating electronic health records with information from sensor systems: considerations relating to standards and architecture arising from the development of a prototype system. Journal of Convergence Information Technology, in press, accepted 1 Oct 2009.

[4] Xiao, S., Dhamdhere, A., Sivaraman, V. \& Burdett, A. 2009, 'Transmission Power Control in Body Area Sensor Networks for Healthcare Monitoring', IEEE: Selected Areas in Communication, vol. 27, no. 1, pp. 37-48.

[5] Anastasi, G., Conti, M., Di Francesco, M. \& Passarella, A. 2009, 'Energy Conservation in wireless sensor networks: A survey', Ad Hoc Networks, vol. 7, pp. 537568.

[6] Miao, G., Himayat, N., Li, Y., \& Swami, A. 2008, 'Cross-Layer Optimisation for Energy-Efficient Wireless Communications: A Survey, Wireless Communications and Mobile Computing, vol. 9, No. 4, pp.529-542

[7] Dhamdhere, A., Sivaraman, V. \& Burdett, A. 2008, 'Experiments in Adaptive Power Control for Truly Wearable Biomedical Sensor Devices', International
Symposium on Parallel and Distributed Processing with Applications, pp. 919-925.

[8] Colvin, D.P. and Lord, C.J. 1991. 'Falls In the Elderly: Detection and Assessment'. Engineering in Medicine and Biology Society. 13: 1938-1939.

[9] C. H. Duney, H. Massoudi, and M. F. Iskander, "Radiofrequency radiation dosimetry handbook," USAF School of Aerospace Medicine, October 1986.

[10] Italian National Research Council, Institute for Applied Physics, "Dielectric properties of body tissues," http://niremf.ifac.cnr.it

[11] C. Gabriel and S. Gabriel, "Compilation of the dielectric properties of body tissues at RF and microwave frequencies," AL/OE-TR-1996-0037, June 1996.

[12] P. Gandhi, ".Biological effects and medical applications of electromagnetic energy," Prentice Hall, Englewood Cliffs, N.J., 1990.

[13] Yingwei Yao and Gerogios B. Giannakis. Energyefficient scheduling for wireless sensor networks. IEEE Transactions on Communications, 53(8):1333-1342, August 2005.

[14] Bhaskar Krishnamachari. Networking Wireless Sensors. Cambridge University Press, 2005.

[15] Ioannis Chatzigiannikis, Athanasios Kinalis, and Sotiris Nikoletseas. Efficient data propagation strategies in wireless sensor networks using a single mobile sink. Computer Communications, 31(5):896-914, March 2008 .

[16] Zhang, J., Chen, J. \& Sun, Y. 2008, 'Transmission Power Adjustment of Wireless Sensor Networks Using Fuzzy Control Algorithm', Wireless Communications and Mobile Computing, vol. 9, no. 6, pp. 805-818.

[17] Lin, S., Zhang, J., Zhou, G., Gu, L., He, T. \& Stankovic, J.A. 2006, 'Adaptive Transmission Power Control for Wireless Sensor Networks', SenSys '06: Proceedings of the 4th international conference on Embedded networked sensor systems, pp. 223-236.

[18] Kubisch, M., Karl, H., Wolisz, A., Zhong, L.C. \& Rabaey, J. 2003, 'Distributed Algorithms for Transmission Power Control in Wireless Sensor Networks', IEEE Wireless Communications and Networking Conference, vol. 1, pp. 558-563.

[19] Lockhart, T. 2003. 'Why do the elderly fall?' Virginia Tech Science Coalition Page, http://www.research.vt.edu/resmag/sc2003/whyfall.htm [Accessed 01/05/2009].

[20] Butler, M., Kerse, N. and Todd, M. 2004. 'Circumstances and consequences of falls in residential care: the New Zealand story'. The New Zealand Medical Journal. 117 (1202).

[21] Hayes, W.C., McMahon, T.A. and Smeesters, C. 2001. 'Disturbance type and gait speed affect fall direction and impact location'. Journal of Biomechanics. 34: 309-317.

[22] Bourke, A.K., Lyons, G.M. and O'Brien, J.V. 2008. 'Evaluation of a threshold-based tri-axial accelerometer fall detection algorithm'. Gait \& Posture. 26: 194-199.

[23] Brown, G. 2005. 'An Accelerometer Based Fall Detector: Development, Experimentation, and Analysis'. University of California, Berkeley.

[24] Haux, R., Lohse, A., Marschollek, M. and Wolf, K.H. 2007. 'Development of a Fall Detector and Classifier based on a Triaxial Accelerometer Demo Board'. Procceedings of the 9th International Conference on Ubiquitous Computing UbiComp, Innsbruck. 
Method for Increasing the Energy Efficiency of Wirelessly Networked Ambulatory Health Monitoring Devices Bruce Moulton, Graham Croucher, Andrew Varis, June Chen

[25] Jämsä, T., Kangas, M., Konttila, A. and Winblad, I. 2007. 'Determination of simple thresholds for accelerometry-based parameters for fall detection'. Engineering in Medicine and Biology Society. 22-26: $1367-1370$. 